\title{
Double-targeting CDCA8 and E2F1 inhibits the growth and migration of malignant glioma
}

\author{
Xiaoxiong Wang $\mathbb{1}^{1,2}$, Heping Wang ${ }^{3}$, Jiajun $\mathrm{Xu}^{1,2}, \mathrm{Xu} \mathrm{Hou}^{1,2}$, Haoqiang $\mathrm{Zhan}^{4}$ and Yunbo Zhen ${ }^{1,2}$
}

\begin{abstract}
High-grade glioma is the most common and aggressive primary brain tumor in adults with poor therapeutic efficiency and survival prognosis. Cell division cycle associated 8 (CDCA8) has been well known as a cell cycle regulator and tumor promotor in various malignant tumors. However, its biological role in glioma still remains unclear. Our results showed that high level of CDCA8 was significantly correlated with advanced WHO grade and poor overall survival and disease-free survival prognosis. In vitro and in vivo investigations demonstrated that CDCA8 promoted the glioma malignancy by promoting cell proliferation, cell migration, and inhibiting cell apoptosis. Moreover, we found its synergetic biological protein-E2F1 by the gene microarray chip. In this study, we revealed that CDCA8 synergized with E2F1 facilitated the proliferation and migration of glioma. In conclusion, our study provides a novel promising therapeutic targets and prognostic biomarkers for malignant glioma treatment.
\end{abstract}

\section{Introduction}

High-grade glioma is the most common malignant primary brain tumor. Despite current advances in treatment for high-grade glioma, the overall prognosis remains poor, and long-term survival is rare ${ }^{1,2}$. To date, the multimodal therapy for malignant gliomas incorporating surgery, radiotherapy, systemic therapy (chemotherapy, targeted therapy), and supportive care improved the therapeutic effect of single therapy ${ }^{3,4}$. However, due to the clinical characteristics of invasive growth, malignant glioma cells migrate outward along the brain white matter, para-vascular spaces, or nerve fibers, making it impossible to be completely removed. Even if the patient undertook the comprehensively therapeutic strategy, these tumor seeds hidden in the cerebral microenvironment niches

\footnotetext{
Correspondence: Haoqiang Zhan (zhanhq@mail.sysu.edu.cn) or

Yunbo Zhen (zhenyb295@163.com)

${ }^{1}$ Department of Neurosurgery, The First Affiliated Hospital of Harbin Medical University, No. 23 Youzheng Street, Nangang District, 150001 Harbin, Heilongjiang Province, People's Republic of China

${ }^{2}$ Institute of Brain Science, Harbin Medical University, No. 23 Youzheng Street, Nangang District, 150001 Harbin, Heilongjiang Province, People's Republic of China

Full list of author information is available at the end of the article These authors contributed equally: Xiaoxiong Wang, Heping Wang Edited by B. Joseph
}

may still cause rapid recurrence, which is the major factor for poor survival of patients with malignant gliomas ${ }^{5,6}$. Approximately $50 \%$ of postoperative gliomas recur with more aggressively malignant biological behaviors and rapidly deteriorative circumstances. Current studies have shown that hypoxic microenvironment, key oncogenetic and metabolic pathways are involved in the initiation and aggression of malignant gliomas ${ }^{6-8}$. The era of precision oncology renders much promises in developing more efficient therapies to confront this aggressive disease. Therefore, the elaboration of the underlying molecular mechanism will provide potential therapeutic targets and prognostic biomarkers for novel glioma therapeutic strategies.

Chromosomal passenger complex (CPC) is the important regulatory structure involved in cell proliferation and division'. CDCA8 (also known as Borealin/DasraB) protein encoded by human cell division cycle associated 8 gene is another major component in the $\mathrm{CPC}$, except INCENP, Survivin, and Aurora $\mathrm{B}^{10}$. $C D C A 8$ as cell cycle regulatory gene plays a key role in recruiting $\mathrm{CPC}$ to centromeres, correcting kinetochore binding errors, and stabilizing double-click spindles ${ }^{11}$. In recent years, increasing studies have shown that overexpressed CPC

\section{(c) The Author(s) 2021}

\footnotetext{
(c) Open Access This article is licensed under a Creative Commons Attribution 4.0 International License, which permits use, sharing, adaptation, distribution and reproduction cc) in any medium or format, as long as you give appropriate credit to the original author(s) and the source, provide a link to the Creative Commons license, and indicate if changes were made. The images or other third party material in this article are included in the article's Creative Commons license, unless indicated otherwise in a credit line to the material. If material is not included in the article's Creative Commons license and your intended use is not permitted by statutory regulation or exceeds the permitted use, you will need to obtain permission directly from the copyright holder. To view a copy of this license, visit http://creativecommons.org/licenses/by/4.0/.
} 
component proteins are responsible for the dysfunction of chromosomal passenger complexes, abnormal cell division, and aneuploidy, thereby accelerating the tumorigenesis and malignancy ${ }^{9,10,12}$. The components of CPC, Survivin, and Aurora B, are overexpressed in various types of cancers, while another two components, CDCA8 and INCENP, are rarely studied in tumors ${ }^{13-15}$. The analysis of CDCA8 expression showed that it was highly expressed in tumor cells and undifferentiated human embryonic stem cells, but no or weakly expressed in normal tissue cells $^{16,17}$. Notably, the biological function of CDCA8 has not been extensively investigated in glioma.

In the present study, we demonstrated the prognostic role of CDCA8 in high-grade glioma. And we further screened target genes of CDCA 8 and investigated the potential role of CDCA8 synergized with the transcription factor E2F1 in glioma cell proliferation and migration, indicating the underlying molecular mechanism of CDCA8/E2F1-associated tumor suppression in glioma treatment.

\section{Materials and methods \\ Cell culture}

Human glioma cell lines U251 and SHG-44 were obtained from the Hangzhou BeNa Technology. SHG-44 and U251 cells were maintained in 90\% DMEM medium (Gibco) supplemented with 10\% FBS. All cells were grown in an incubator at $37^{\circ} \mathrm{C}$ with $5 \% \mathrm{CO}_{2}$.

\section{Immunohistochemistry analysis (IHC)}

Glioma tissue and adjacent normal tissue microarray (Cat. \#HBraG180Su01, Shanghai Outdo Biotech Company) was applied for IHC analysis. Before IHC assay, the microarrays were baked at $65^{\circ} \mathrm{C}$ for $30 \mathrm{~min}$. Next, microarrays were dewaxed in xylene and hydrated in ethanol with different concentrations. Citrate buffer was used for antigen repairing at $180^{\circ} \mathrm{C}$ for $5 \mathrm{~min}$. After blocked with $3 \% \mathrm{H}_{2} \mathrm{O}_{2}$, anti-CDCA8 was incubated with the sections at $4{ }^{\circ} \mathrm{C}$ overnight. Secondary antibody was added and incubated for $2 \mathrm{~h}$ at room temperature. Finally, the tissue microarrays were stained with diaminobenzidine and analyzed using CaseViewer 2.0 and Image Scope. The IHC scoring of specimens was determined by the staining intensity and extent scores, which graded as 0 (0\%), 1 (1-25\%), 2 (26-50\%), 3 (51-75\%), or 4 (76-100\%). Antibodies used were shown in Table S1.

\section{Plasmid construction, lentivirus infection, and transfection} shRNA sequences of human gene CDCA8 and E2F1 were designed at Shanghai Yibeirui Biomedical Science and Technology Co., Ltd (sequences for CDCA8 and E2F1 were detailed in Table S2). Target shRNA sequences were inserted into BR-V-108 vectors, and then transformed into E. coli competent cells (Tiangen) for plasmid extraction using EndoFree maxi plasmid kit (Tiangen). Qualified plasmids were packaged for lentivirus production using 293T cells which were co-transfected with BRV-108, pHelper 1.0, and 2.0 Vector with Lipofectamine 2000 reagent (Thermo Fisher Scientific). Lentiviral vector containing amplification sequence of CDCA8 was also constructed for overexpression. Human glioma cell lines stably expressing CDCA8 and E2F1 protein were established by transfecting the BR-V-108-CDCA8 or BR-V108-E2F1lentivirus into SHG-44 and U251 cells at $80 \%$ confluence using Lipofectamine 2000 reagent (Thermo Fisher Scientific). After $72 \mathrm{~h}$, cell infection efficiency was evaluated by microscopic fluorescence. Lentivirus transfected cells were prepared for the next experiment.

\section{RNA extraction and RT-qPCR}

Lentivirus transfected cells at $80 \%$ confluence were harvested and total RNA was extracted using Trizol reagent (Invitrogen). The quality of total RNA was evaluated by Nanodrop 2000 spectrophotometer (Thermo Fisher Scientific) according to the manufacturer's instructions. $2.0 \mu \mathrm{g}$ total RNA was reverse transcribed into cDNA using Promega M-MLV. 1.0 $\mu \mathrm{L}$ cDNA was added into the reaction system for real-time PCR using SYBR Green master mix Kit (TAKARA) on the platform of Sequence Detection system (TAKARA). The related primers used were shown in Table S3. The relative quantitative analysis in gene expression, data were analyzed by the $2^{-\Delta \Delta C t}$ method with GAPDH as inner control.

\section{Western blotting assay (WB)}

Lentivirus transfected cells at $80 \%$ confluence were harvested and lysed in ice-cold lysis buffer, and the total protein collected and the protein concentration was detected by BCA Protein Assay Kit (HyClone Pierce). Equal amount proteins $(20 \mu \mathrm{g})$ were separated by $10 \%$ SDS-PAGE. After all, proteins were transferred onto PVDF membrane, the membrane was blocked at room temperature for $1 \mathrm{~h}$ with $5 \%$ non-fat milk TBST solution. Then the blocked membrane was incubated with primary antibodies at $4{ }^{\circ} \mathrm{C}$ overnight and continuingly incubated with the secondary antibody for $2 \mathrm{~h}$ at room temperature. The outcomes were visualized by ECL plus TM Western blotting system kit (Amersham). Gray scanning was analyzed by Image J. Antibodies used in WB were detailed in Table S1.

\section{MTT assay}

Lentivirus transfected cells in exponential growth phase were trypsinized and seeded into a 96-well plate (2000 cells/well) and cultured. In all, $20 \mu \mathrm{L}$ of $5 \mathrm{mg} / \mathrm{mL}$ MTT solution (Sangon Biotech) was added at 1st, 2nd, 3rd, 4th, and 5 th day, respectively. After reaction for $4 \mathrm{~h}, 100 \mu \mathrm{L}$ 
DMSO solution was added and the absorbance values at $490 \mathrm{~nm}$ were measured by a microplate reader (Tecan). Cell viability was detected by MTT assay according to the manufacturer's protocol.

\section{Flow cytometry assay}

Apoptosis and cell cycle were detected by flow cytometry assay. Lentivirus transfected cells were inoculated in 6-well dishes with $5 \mathrm{~mL}$ per well $\left(1 \times 10^{3}\right.$ cells $\left./ \mathrm{mL}\right)$ in triplicate and cultured until the confluence reached $70 \%$. Cells were collected and washed with $4{ }^{\circ} \mathrm{C}$ D-Hanks, then centrifugated $(1300 \mathrm{rmp})$. Cells were resuspended with $200 \mu \mathrm{L} 1 \times$ binding buffer, and stained by $10 \mu \mathrm{L}$ Annexin V-APC (eBioscience) in the dark. For cell cycle detection, cells were dyed by PI (Sigma) staining solution, then cells were detected using Millipore flow cytometry.

\section{Colony formation assay}

Cells in the logarithmic growth phase were trypsinized, resuspended, and seeded into 6 -well plates at 600 cells per well in triplicate. After cultured for 8 days, cell clones were photographed under a fluorescence microscope (Olympus). Then all clones were fixed by $4 \%$ paraformaldehyde (SIGMA) and stained by Giemsa. Finally, clones were washed with $\mathrm{ddH}_{2} \mathrm{O}$ several times, dried, and photographed with a digital camera. Colony-forming rate $=($ colony number $/$ inoculated cell number $) \times 100 \%$.

\section{Wound-healing assay}

Lentivirus transfected cells were seeded at $3 \times 10^{4}$ cells per well onto a 96-well dish. Scratches were made by a 96wounding replicator (VP scientific) across the cell layers while the cell confluence reached over $90 \%$. Serum-free medium was used to rinse gently 2-3 times and floating cells were washed. RPMI-1640 medium with $0.5 \%$ FBS was added and cultured for $24 \mathrm{~h}$ and photographs were taken by a fluorescence microscope at 8 and $24 \mathrm{~h}$. Cell migration rate of each group was calculated.

\section{Transwell assay}

Transwell assay was performed by Corning Transwell Kit. First, transfected SHG-44 and U251 cells were collected, trypsinized, counted, and incubated in the upper chamber with $100 \mu \mathrm{L}$ medium without FBS in a 24-well plate $\left(1 \times 10^{6}\right.$ cells/well). Six hundred microliters medium supplemented with $30 \%$ FBS was added in the lower chamber. Cells were incubated for $48 \mathrm{~h}$ and then non-metastatic cells were removed with a cotton swab. Cells were fixed and stained by $4 \%$ formaldehyde and Giemsa, respectively, then the migration ability of cells was analyzed.

\section{Cell counting assay}

Transfected cells were seeded into 96-well plates (2000 cells/well) and further cultured in DMEM supplemented with $10 \% \mathrm{FBS}$ at $37^{\circ} \mathrm{C}$ with $5 \% \mathrm{CO}_{2}$ for 5 days. Medium was changed every three days. Celigo image cytometer (Nexcelom Bioscience) was applied for cell counting on days $1,2,3,4,5$ and the data were analyzed.

\section{Co-immunoprecipitation (Co-IP) assay}

Total protein from lentivirus transfected SHG-44 cells expressing CDCA8 were collected and Co-IP assay was applied for identifying whether CDCA8 and E2F1 interact with each other. $1.0 \mathrm{mg}$ total protein was incubated with anti-DYKDDDDK Tag (binds to the same epitope as Sigma's Anti-FLAG® M2 Antibody), anti-HA, antiCDCA8, and anti-E2F1 at $4{ }^{\circ} \mathrm{C}$ overnight. Twenty microliters agarose beads were added and incubated at $4{ }^{\circ} \mathrm{C}$ for $2 \mathrm{~h}$. After centrifugation at $2000 \times g$ for $1 \mathrm{~min}$, supernatant was discarded. Protein A/G beads were collected and washed twice. Next, the Protein A/G beads were denatured in the IP lysate buffer and $5 \times$ loading buffer at $100{ }^{\circ} \mathrm{C}$ boiling water for $5 \mathrm{~min}$. Finally, $20 \mu \mathrm{g}$ protein sample was subjected WB analysis described in WB assay section.

\section{Microarray assay}

Total RNA was extracted by the RNeasy kit (Sigma). Concentration and values of A260 and A280 of total RNA were determined by Nanodrop 2000 (Thermo Fisher Scientific). RIN value was evaluated with Agilent 2100andAgilent RNA 6000 Nano Kit. RNA sequencing was performed with Affymetrix human Gene Chip PrimeView according to the manufacturer's instruction and the outcomes were scanned by Affymetrix Scanner 3000 (Affymetrix). Gene expression in SHG-44 cells transfected with shCDCA8 or shCtrl was analyzed with PrimeView Human Gene Expression Array (Affymetrix) and the microarray raw data were generated, statistical significance assessment was accomplished using a Welch $t$ test with Benjamini-Hochberg FDR ( $\log 2$ fold change $>1.3$ and FDR $<0.05$ as significant). Significant difference genes bioinformatics analysis based on Ingenuity Pathway Analysis (IPA) (Qiagen) was executed, and $\mid \mathrm{Z}$-score $\mid>2$ is considered meaningful.

\section{Human apoptosis antibody array}

Detection of related genes in human apoptosis signaling pathway was performed using Human Apoptosis Antibody Array (Abcam, ab134001) that simultaneously detects 43 Human Apoptosis marker concentrations in cell and tissue lysates. According to the manufacturer's instructions, the SHG-44 cells expressing CDCA8 were lysed and total proteins were extracted. Protein concentrations were measured by BCA Protein Assay Kit (HyClone Pierce). Antibody array ships were incubated with protein samples $(0.5 \mathrm{mg} / \mathrm{mL})$ overnight at $4{ }^{\circ} \mathrm{C}$ and 
continuing incubated with cocktail of biotin-conjugated antibodies overnight at $4{ }^{\circ} \mathrm{C}$. Next incubated with labeled streptavidin for $2 \mathrm{~h}$. Enhanced chemiluminescence was used for visualizing and spots gray value was analyzed by Image J.

\section{Tumor transplantation model}

Female BALB/c nude mice were purchased from Beijing Vitalriver Experimental Animal Technology Co., Ltd and housed in a $12 \mathrm{~h} \mathrm{light/dark}$ cycle controlled condition at $24^{\circ} \mathrm{C}$. $0.2 \mathrm{~mL}\left(4 \times 10^{6}\right.$ cells $/$ mice $)$ lentivirus transfected SHG-44 cell suspension was subcutaneously injected into 4-week-old mice (5 mice per group, group as $\mathrm{NC}$ vs CDCA8, NC vs shE2F1, NC (KD + OE) vs CDCA8 + shE2F1; or 10 mice per group, group as NC and KD). The lengths $(L)$ and widths $(W)$ of tumor was record and tumor volume later using $L$ and $W$ ( $L$ represent longest dimension and $W$ means dimension perpendicular to length) and calculated as $\pi / 6 \times L \times W^{2}$. Tumor volume was calculated 2-3 times weekly. Fluorescence images were observed by IVIS Spectrum Imaging System (Perkin Elmer). At the end of animal experiment, all mice were sacrificed and tumor tissues were removed for Ki67 immunostaining. All animal experiments conformed to the European Parliament Directive (2010/63/EU) and were approved by the Institutional Animal Care and Use Committee at Harbin Medical University (No. HMUIRB2008-06) and the Institute of Laboratory Animal Science of China (A5655-01).

\section{Ki67 immunostaining}

For Ki67 immunostaining, tumor tissues were fixed in $4 \%$ paraformaldehyde for $24 \mathrm{~h}$, and then were embedded in paraffin for cutting $(4 \mu \mathrm{m})$. After deparaffinization, rehydration, and blocking with $\mathrm{PBS}-\mathrm{H}_{2} \mathrm{O}_{2}$, all sections were incubated with $\mathrm{Ki} 67$ primary antibody at $4{ }^{\circ} \mathrm{C}$ overnight. After washing, sections were further incubated with the secondary antibody, antibodies were detailed in Table S1. Immunostaining was applied by Hematoxylin and Eosin (Baso, Zhuhai, Guangdong, China).

\section{Surgically excised specimens of glioma patients}

A total of 176 glioma patients were enrolled in the Department of Neurosurgery at The First Affiliated Hospital of Harbin Medical University from 2017 to 2019. The mean age of patients was 41 years (range, 8-80y); $62 \%$ were male. The median preoperative KPS score was 70. None of the patients received preoperative radiotherapy or chemotherapy. The tissue samples were routinely collected for histologic diagnosis in strict accordance with WHO criteria, while adjacent specimens were immediately snap-frozen in liquid nitrogen and then stored at $-80^{\circ} \mathrm{C}$ until further analysis. Normal brain tissues were obtained from normal adjacent tissues away from tumor tissues or nonneoplastic brain diseases and were histologically confirmed to be free of any pathological lesions. The study was approved by the Ethics committees at the Harbin Medical University, and all subjects gave informed consent.

\section{Statistical analysis}

Data from independent experiments are shown as the means \pm standard deviations (SD). Statistical analysis between two groups was performed by Student's $t$-test (two-tailed) and among multiple groups was conducted by one-way ANOVA with SPSS version 17.0 (IBM Analytics, USA). CDCA8 expression difference between glioma tissue and adjacent normal tissue was analyzed with Rank Sum test analysis. The relationship of CDCA8 expression and tumor characteristics in glioma patients were analyzed with Mann-Whitney $U$ analysis and Spearman Rank correlation analysis. Survival data were evaluated by using Kaplan-Meier survival analysis. A $P$ value $<0.05$ was considered statistically significant $(*)$.

\section{Results \\ High expression of CDCA8 in glioma predicts poor prognosis}

To determine whether the CDCA8 expression is correlated with the malignant progression and clinical outcomes of gliomas, we detected the CDCA8 level in glioma IHC samples and evaluated the overall survival and disease-free survival outcomes by Kaplan-Meier survival analysis. As shown in Table 1 and Fig. 1A, the expression of CDCA8 was generally higher in glioma tissues. Moreover, the higher level of CDCA8 is associated with advanced WHO grade and tumor recurrence (Table 2 and Table S4). Kaplan-Meier survival analysis showed that patients with relatively higher CDCA8 expression suffer from lower overall survival rate and disease-free survival rate (Fig. 1B). These results indicate that CDCA 8 may be a prognostic biomarker for glioma malignancy and poorer prognosis.

\begin{tabular}{|c|c|c|c|c|}
\hline \multirow[t]{2}{*}{ CDCA8 expression } & \multicolumn{2}{|c|}{ Tumor tissue } & \multicolumn{2}{|c|}{ Normal tissue } \\
\hline & Cases & Percentage & Cases & Percentage \\
\hline Low & 100 & $56.8 \%$ & 24 & $100 \%$ \\
\hline High & 76 & $43.2 \%$ & 0 & $-\%$ \\
\hline
\end{tabular}

$P<0.001$. 


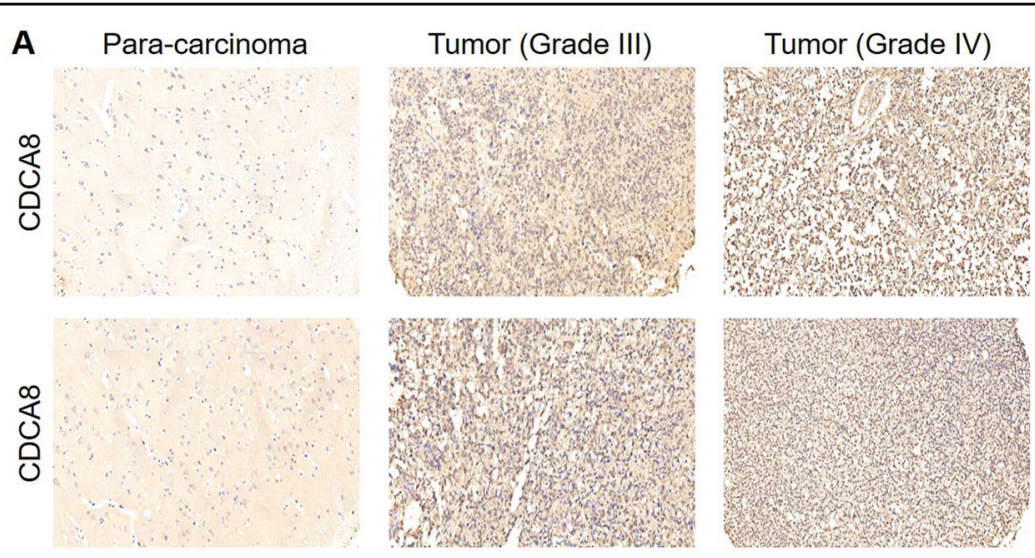

B $\quad$ Log rank $\mathrm{P}<0.001$
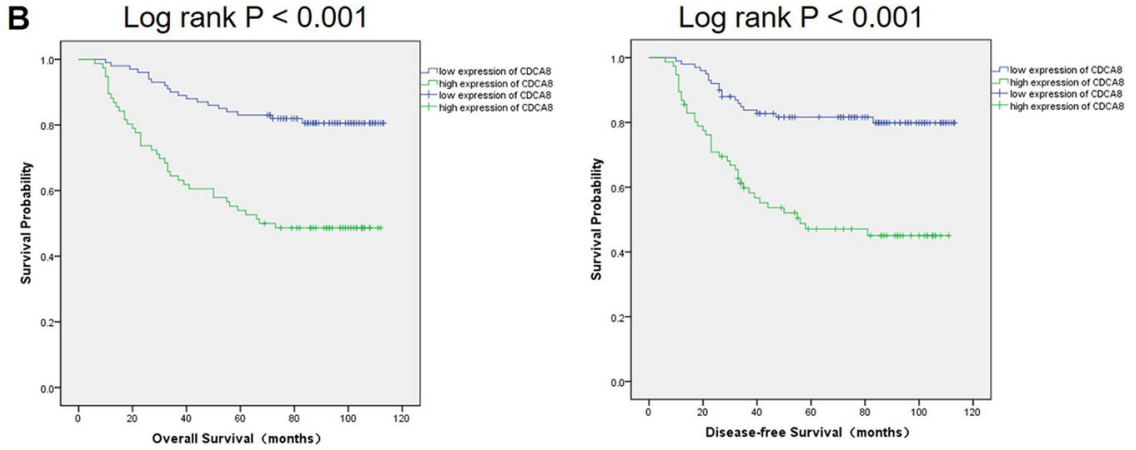

Fig. 1 High expression of CDCA8 in glioma predicts poor prognosis. A CDCA8 expression in para-carcinoma tissues and glioma tissues with different tumor grade. B Kaplan-Meier survival analysis of CDCA8 expression in glioma patients.

Table 2 Relationship between CDCA8 expression and tumor characteristics in patients with glioma.

\begin{tabular}{lcccc}
\hline Features & No. of patients & \multicolumn{2}{l}{$\begin{array}{l}\text { CDCA8 } \\
\text { expression }\end{array}$} & P value \\
\cline { 3 - 4 } & & Low & High & \\
\hline All patients & 176 & 100 & 76 & \\
Age (years) & & 55 & 37 & \\
S41 & 92 & 44 & 39 & \\
$>41$ & 83 & & & 0.368 \\
Gender & & 62 & 47 & \\
Male & 109 & 38 & 29 & \\
Female & 67 & & & $<0.001$ \\
Relapse state & & 39 & 54 & \\
Yes & 93 & 61 & 22 & \\
No & 83 & & & $<0.001$ \\
Grade & & 19 & 5 & \\
I & 24 & 52 & 25 & \\
II & 77 & 24 & 27 & \\
III & 51 & 5 & 19 & \\
IV & 24 & & & \\
\hline
\end{tabular}

CDCA8 promotes glioma cell proliferation and migration in vitro

To assess the effect of CDCA8 on glioma cell viability, apoptosis, cycle distribution, and migration, we used lentivirus vector to silence the CDCA 8 expression in SHG-44 and U251 cells. The derived fluorescence in $>80 \%$ of cells proved the successful infection (Fig. S1A), and the significantly downregulated level of CDCA8 in SHG-44 and U251 cells (Fig. S1B). As shown in Fig. 2A, B, CDCA8 knockdown inhibited glioma cell proliferation $(P<0.001)$ and induced glioma cell apoptosis $(P<0.001)$. Moreover, knockdown of CDCA8 induced the decreased cell population in $S$ phase and increased in $G 2$ phase $(P<0.01$, Fig. $2 \mathrm{C})$. We found that anti-apoptosis proteins $\mathrm{Bcl}-2, \mathrm{Bcl}-\mathrm{w}$, cIAP-2, HSP70, and IGFBP-2 were downregulated, and pro-apoptosis proteins Caspase3, Caspase8, p21, and p27 were upregulated after CDCA8 knockdown (Fig. S2). Furthermore, cell migration was also evaluated by Transwell assays and wound healing, which showed glioma cell migration capacity was suppressed after infected with shCDCA8 (Fig. 2D, E). All these results implied the carcinogenic effect of CDCA8 on glioma progression, which was consistent with our previous results.

\section{Knockdown of CDCA8 inhibited tumorigenesis and glioma growth in vivo}

To investigate the efficacy of CDCA8 knockdown therapy in glioma xenograft model. Interestingly, no 


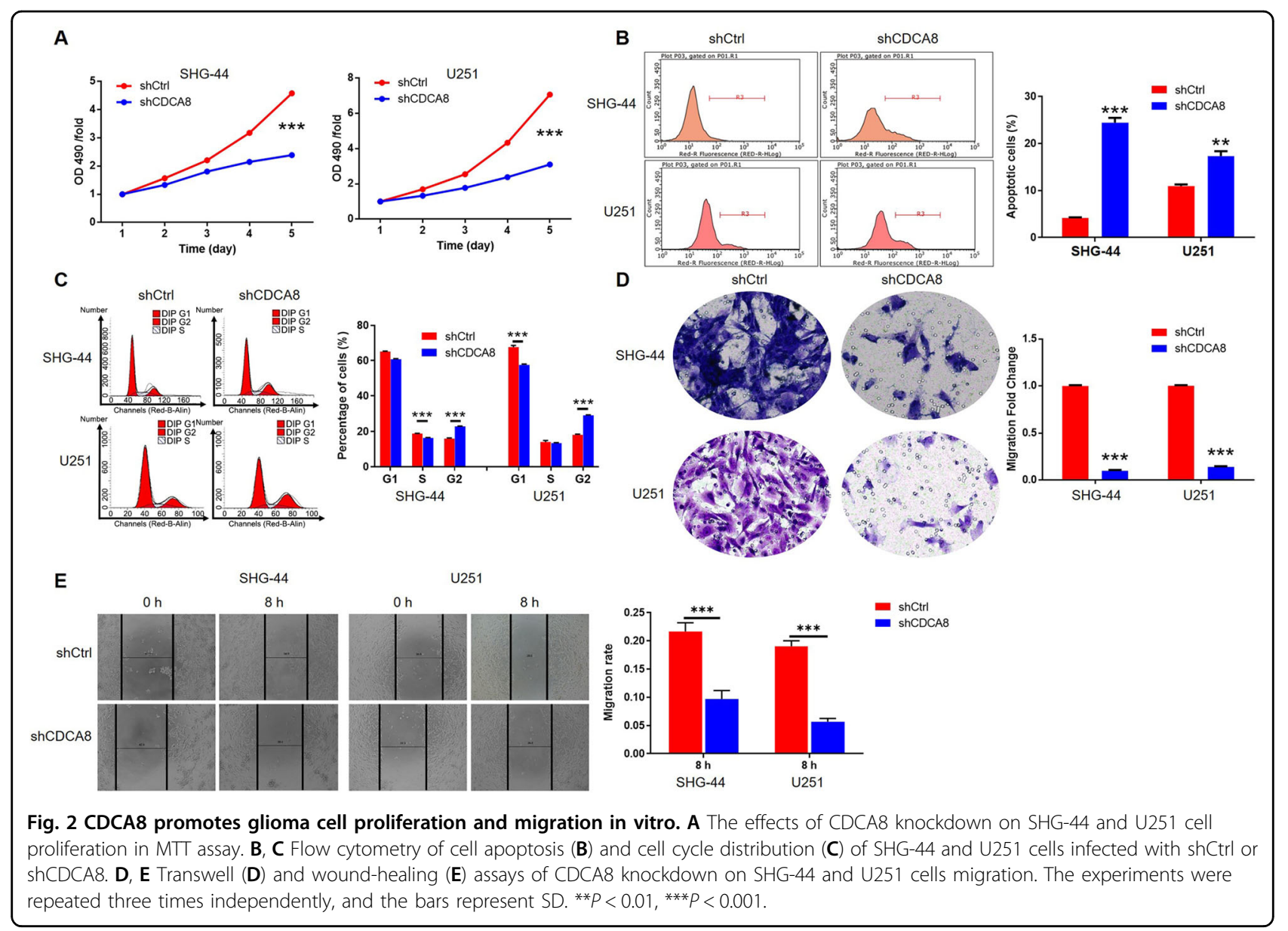

obvious tumors were observed in shCDCA8 U251 group, indicating the weak tumorigenesis treated by CDCA8 knockdown (Fig. 3A). The slower growth rate, smaller tumor weight, and lower density of lentivirus vector derived fluorescence formed in shCDCA8 group suggested the restrained progression of glioma in vivo (Fig. $3 \mathrm{~B}-\mathrm{D})$. Ki67, commonly used as a parameter of proliferative activity of tumors, was detected in all xenografts and found to be downregulated in CDCA8 knockdown group (Fig. 3E). Altogether, CDCA8 knockdown exhibits anti-glioma properties in vivo.

CDCA8 synergized with E2F1 as the coregulators in glioma

To further explore the underlying mechanism of CDCA8 in glioma progression, a microarray analysis was performed to identify the potential genes regulated by CDCA8. In a total of 299 upregulated and 663 downregulated genes were found following the criteria: $\log 2$ fold change $>1.3$ and FDR $<0.05$ (Figs. 4A and S3A). Then, several enriched signaling pathways such as HIPPO and interferon signaling pathways (Figure S3B) and disease and functions such as cancer (Figure S3C) were distinguished to construct the CDCA8-related interaction network (Fig. 4B). Subsequently, several candidates were selected based on the network and subjected further by qPCR and western blotting. As shown in Figs. 4C and S3D, co-expression pattern of CDCA8 and E2F1 was clearly displayed. Moreover, the results of co-IP assay indicated that CDCA8 and E2F1 may act as coregulators in glioma malignancy (Fig. 4D).

\section{CDCA8/E2F1 axis regulates glioma progression in vitro and in vivo}

To investigate whether CDCA8 regulates glioma development through E2F1, overexpressed CDCA8, and silenced E2F1 lentiviruses were constructed for U251 cells infection. The efficiencies of infection, CDCA8 overexpression, and E2F1 knockdown were shown in Fig. S4, indicating the alleviated CDCA8 overexpression and E2F1 knockdown in CDCA8 + shE2F1 group compared with the single interference group. This phenotype demonstrated that overexpression of CDCA8 promoted glioma cell proliferation $(P<0.01$, Fig. $5 \mathrm{~A})$ and migration $(P<$ 0.001 , Fig. 5B, C), while inhibiting cell apoptosis $(P<0.01$, Fig. 5 C). Adverse results were obtained in E2F1 knockdown cells $(P<0.01$, Fig. $5 \mathrm{~A}-\mathrm{D})$. More than that, 
A

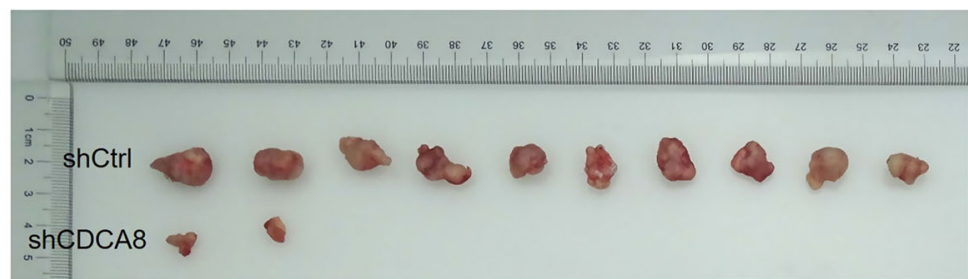

B
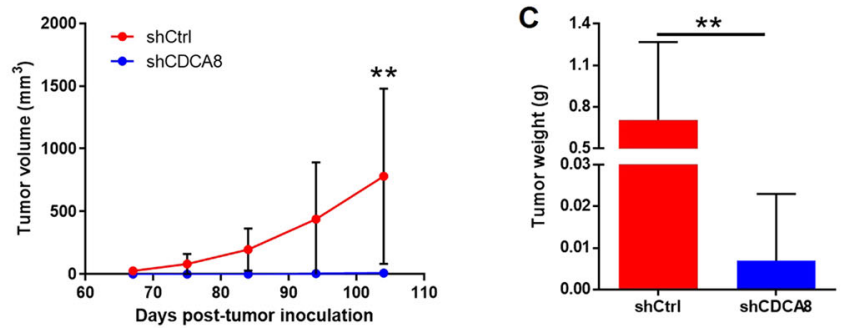

D

shCtrl

shCDCA8
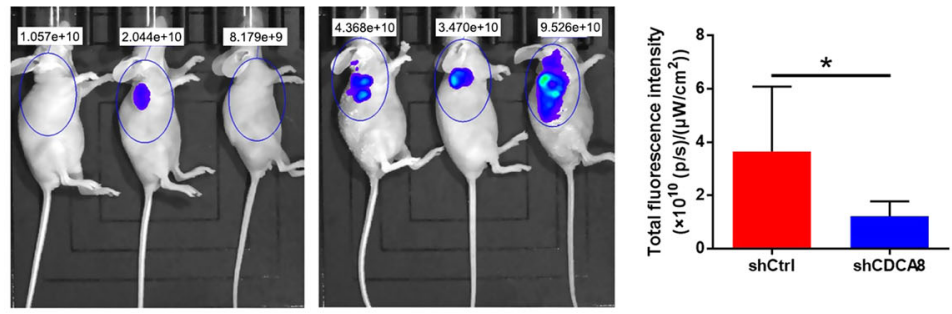

E

shCtrl

shCDCA8

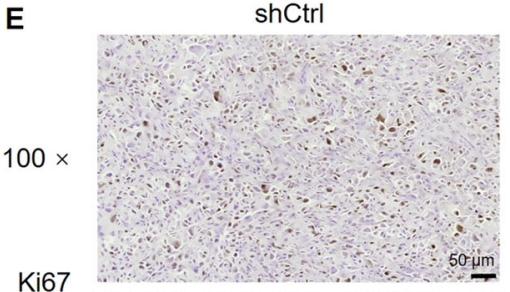

Ki67

$200 \times$
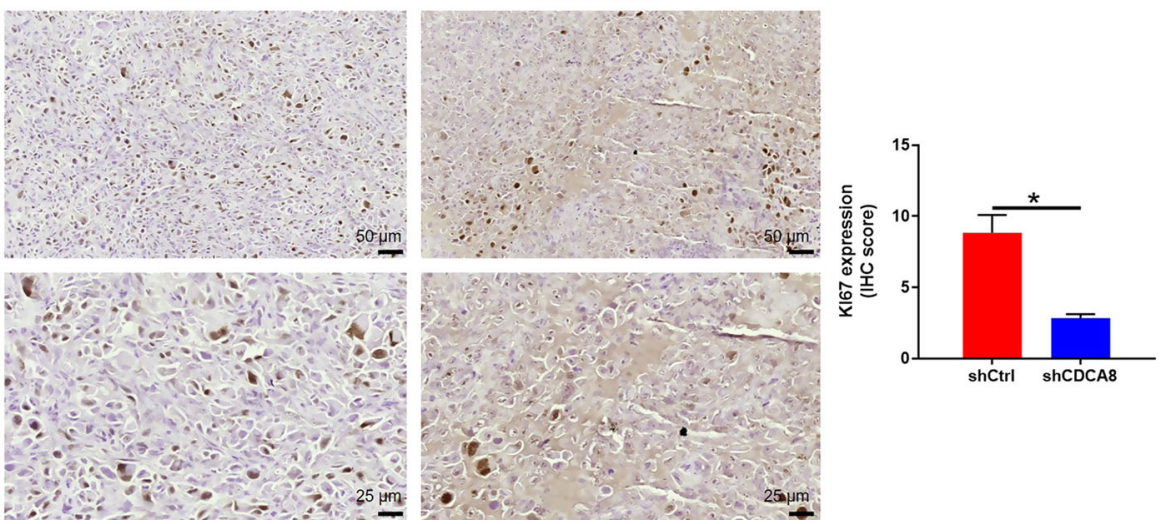

Fig. 3 Knockdown of CDCA8 inhibited tumorigenesis and glioma growth in vivo. Mice xenograft models were constructed by subcutaneously injecting U251 cells. A Dissected tumors from xenograft models with or without shCDCA8 after implantation were collected for taking photos. B Tumor growth curve for tumor volume calculation (from 67 to 104 days post injection). C Tumor weight of mice xenograft model analysis was measured after tumor collection. D In vivo fluorescence imaging of tumor burden was performed just before sacrificing the mice. E Ki67 level by IHC analysis in tumor sections was detected after the tumor collection. Data were performed by mean with SD. ${ }^{*} P<0.05,{ }^{* *} P<0.01$.

outcomes of CDCA8 group, shE2F1 group, and CDCA $8+$ shE2F1 groups demonstrated that the carcinogenic effect of CDCA8 were generally reversed by simultaneous E2F1 knockdown in vitro and vivo $(P<0.01$, Fig. 5A-D). Although not statistically significant, it should be observed that CDCA8 promoted tumor growth in vivo (Fig. S5). Subsequently, we further illustrated weaker fluorescence intensity $(P<0.05)$, slower tumor growth
$(P<0.05)$, and lighter tumor weight $(P<0.05)$ in both shE2F1 and CDCA $8+$ shE2F1 groups in comparison with the respective control group (Figs. 6A, B and S6A-B), which was consistent with Ki67 expression in IHC (Fig. 6C). Notably, the inhibition of tumor growth in CDCA $8+$ shE2F1 group was relatively weaker than that in shE2F1 group. These results indicated the synergistic effects of CDCA8 and E2F1 on the progression of glioma. 


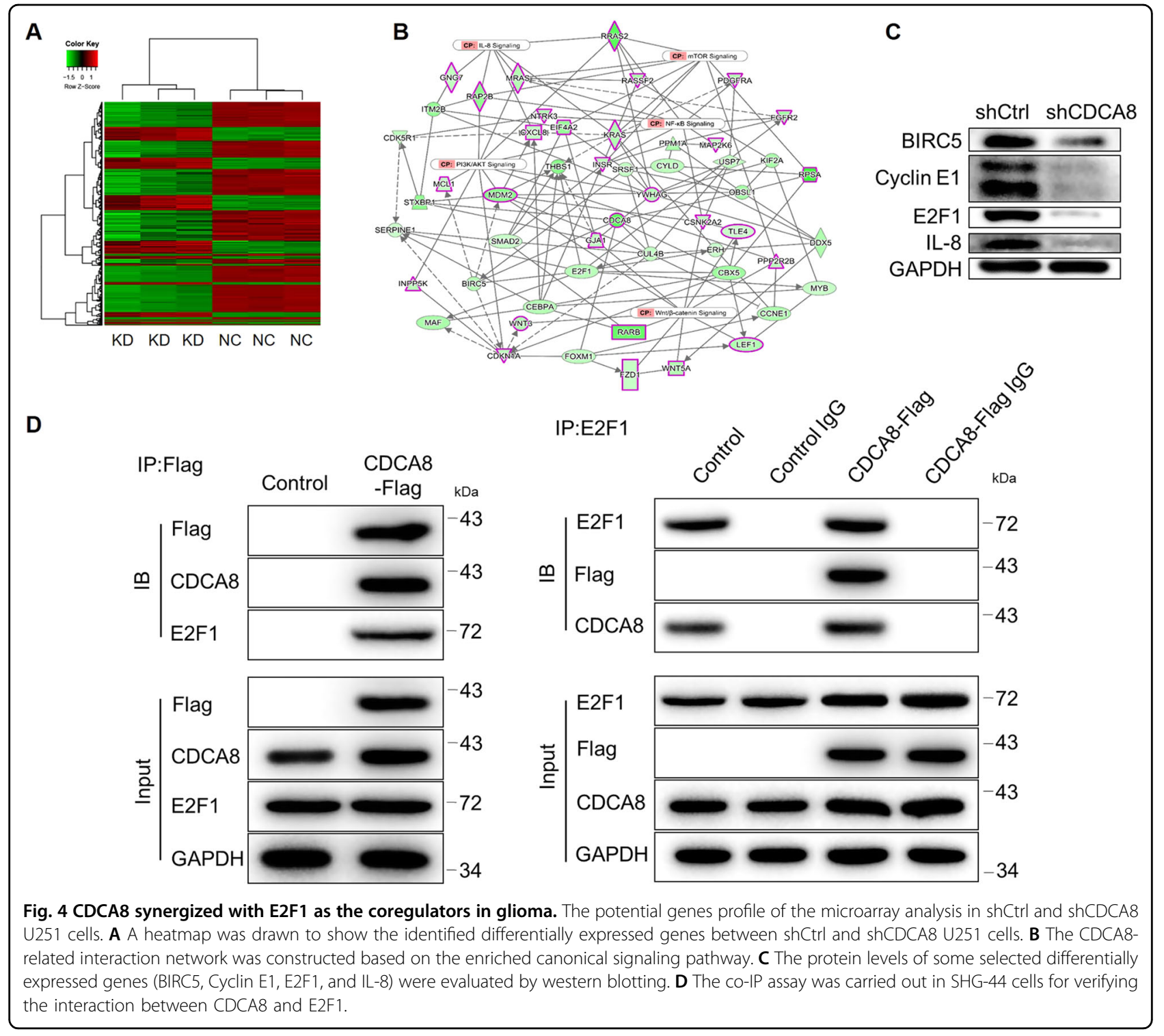

\section{Discussion}

Clinically relevant tissue-based biomarkers of gliomas may help neurosurgeons to further classify gliomas with different biological characteristics, improve survival prognosis, and provide therapeutic targets ${ }^{18,19}$. In this study, we demonstrated that high expression of CDCA8 was associated with advanced WHO grade III-IV and poor overall and disease-free survival, indicating CDCA8 may be a biomarker for the high-grade glioma patients' disease progression and prognosis. Moreover, CDCA8 exhibited its carcinogenetic role in glioma cell growth and migration in vitro and vivo, silence of which could be a promising strategy to suppress glioma progression.

CDCA8 as the main component of CPC is responsible for accurately controlling the normal separation of the cell nucleus and the normal cellular division ${ }^{20}$. CDCA8 is highly expressed in human embryonic stem cells and may have an important role in maintaining stem cell totipotency ${ }^{21}$. Increasing anti-cancer drug researches focus on $\mathrm{CPC}$, the basic regulator, in cell division. Accumulating evidence showed the upregulation of CDCA8 in human cancers and highlighted its biological functions in tumorigenesis. In 2007, Hayama et al. reported the phosphorylation-mediated regulation of CDCA8 by another $\mathrm{CPC}$ component Aurora $\mathrm{B}$, thus acting as tumor promotors in human lung cancer ${ }^{22}$. Ci et al. investigated the role of CDCA8 in malignant melanoma, indicating that CDCA8 could promote cell proliferation, migration, and invasion of melanoma cells through suppressing ROCK signaling pathway and predict poor prognosis of patients $^{23}$. Similar CDCA8 functions in the estrogenstimulated development and progression of breast cancer 


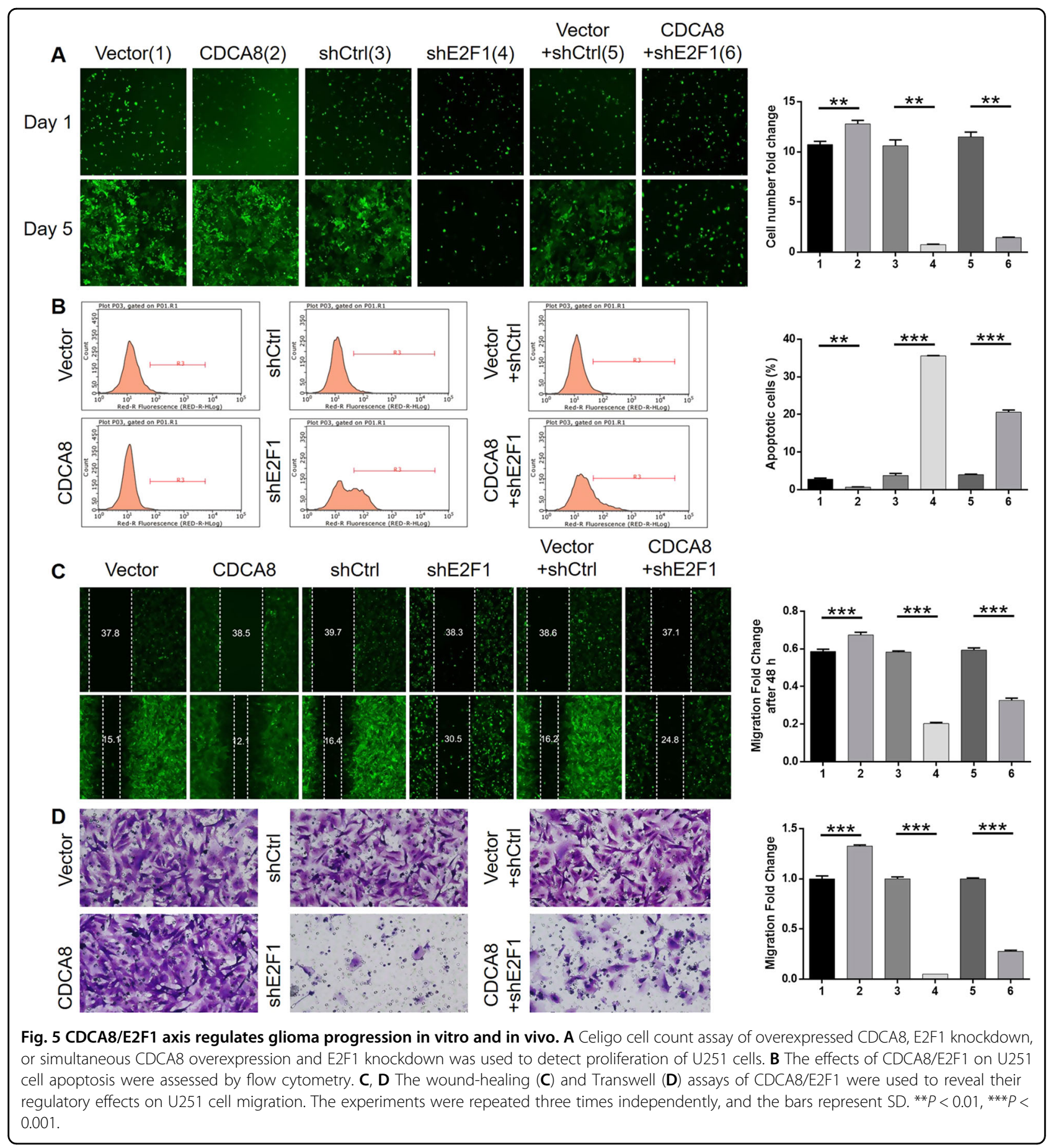

were also revealed by $\mathrm{Bu}$ et $\mathrm{al}^{24}$. Notably, our study is consistent with $\mathrm{Bu}$ et al.'s study, which showed CDCA8 regulated apoptosis-related proteins including Bcl-2, p21, and $\mathrm{p} 27^{24}$. Yu et al. further pointed out that the expression of CDCA8 in breast cancer is associated with tamoxifen resistance ${ }^{25}$. And Dai et al. recognized CDCA8 as a downstream target of transcriptional activation by transcription factor NF-Y in both human embryonic stem cells and cancer cells, demonstrating the potential regulatory mechanism of $\mathrm{CDCA} 8^{21}$. Besides, abnormal expression and positive regulatory functions of CDCA8 in clear cell renal cell carcinoma and bladder cancer have also been illuminated ${ }^{26,27}$. In our study, we found that CDCA8 promoted glioma cell proliferation by inhibiting cell apoptosis and cell cycle arrest, and enhanced cell migration. Subsequent microarray analysis revealed the 
A
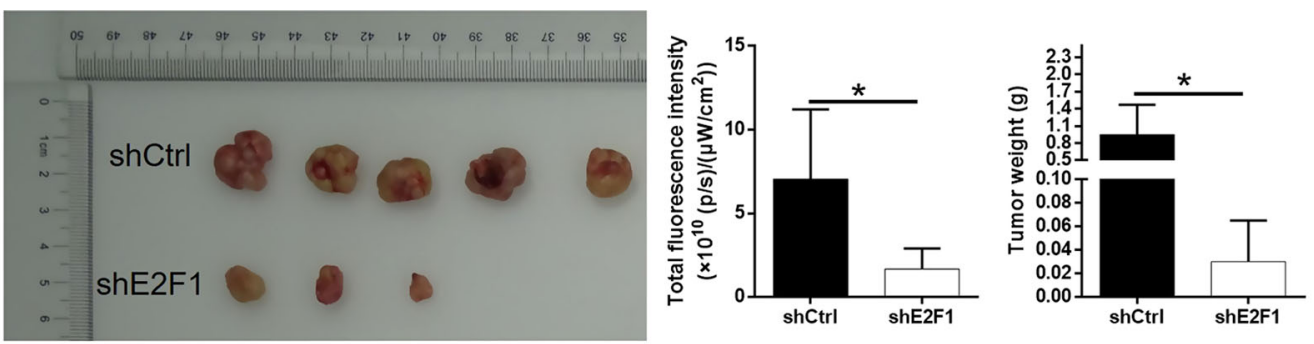

B
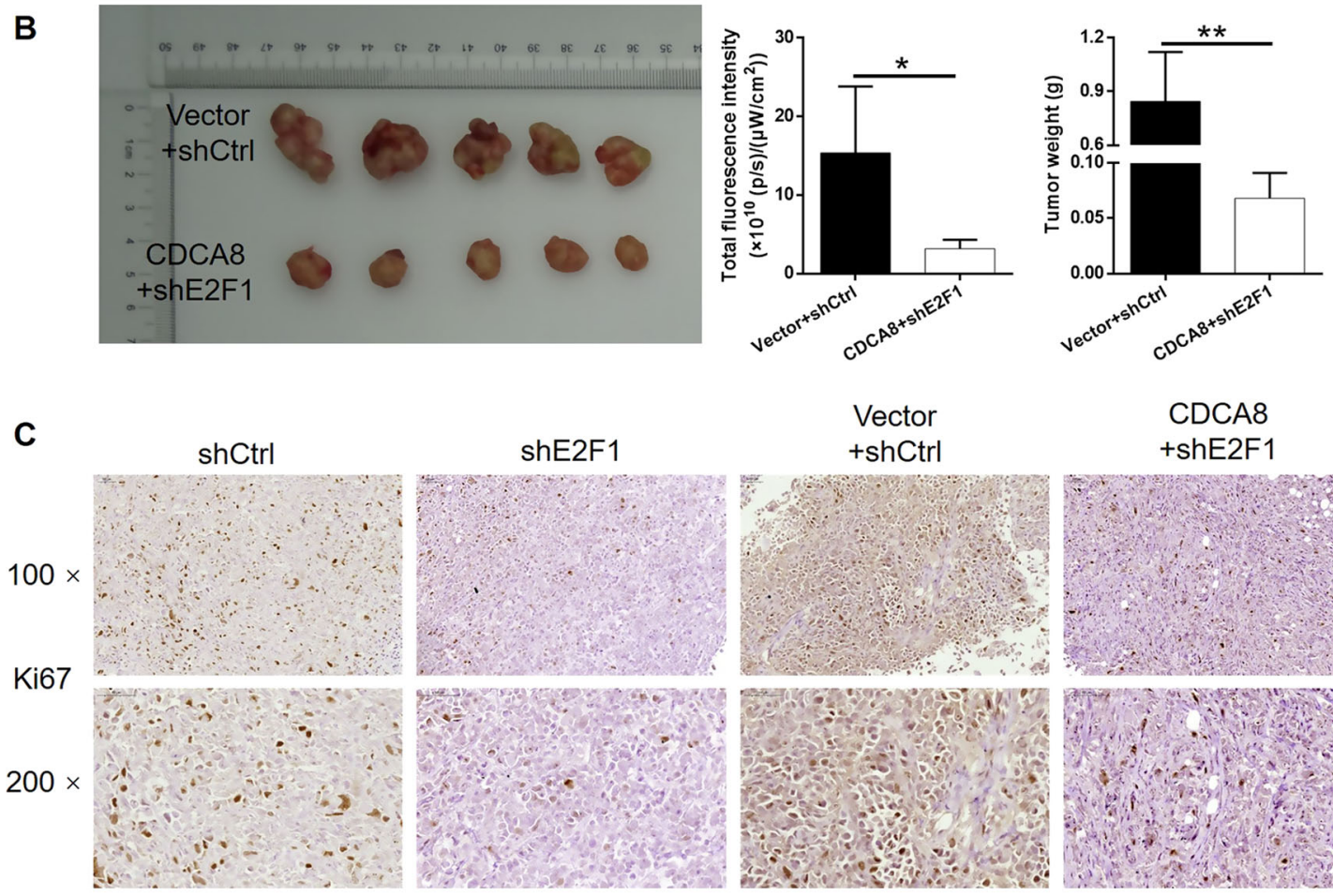

ShE2F1
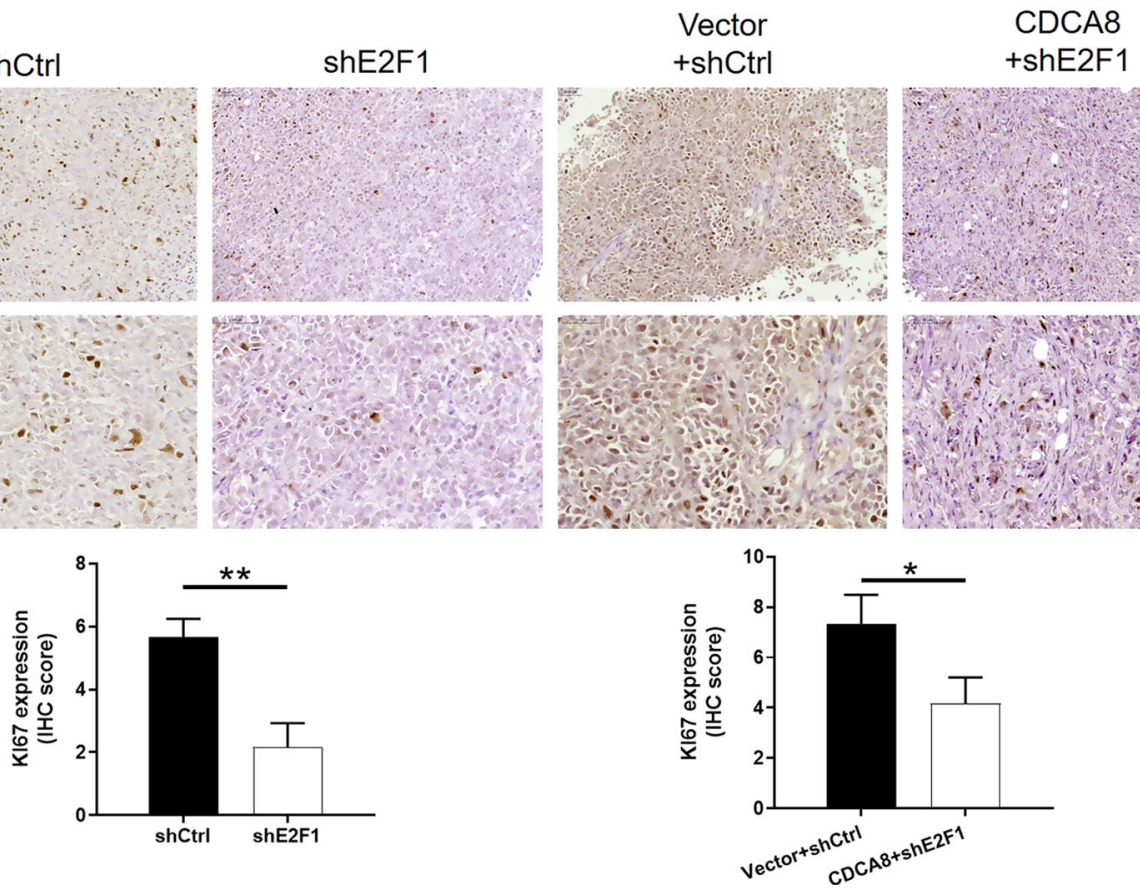

Fig. 6 CDCA8/E2F1 axis regulates glioma progression in vitro and in vivo. Mice xenograft models were constructed through subcutaneously injecting U251 cells in shCtrl, shE2F1, Vector+ shCtrl, and CDCA8 + shE2F1 groups. A, B Dissected tumors from xenograft models with or without shCDCA8 after implantation were collected for taking photos. In vivo fluorescence imaging of tumor burden was performed just before sacrificing the mice. Tumor weight of mice xenograft model analysis was measured after tumor collection. C Ki67 level by IHC analysis in tumor sections was detected after the tumor collection. Data were performed by mean with SD. ${ }^{*} P<0.05,{ }^{* *} P<0.01$.

potential target genes regulated by CDCA8. Meanwhile, combining with the analysis of CDCA8-related interaction network, we focus on E2F1 as the CDCA8 regulatory protein.

E2F1 is a member of the transcription factors E2Fs family involved in apoptosis and DNA damage response. E2Fs are downstream effectors of the tumor suppressor retinoblastoma gene product $\mathrm{pRb}$ and play a role in multiple cytological behaviors such as DNA synthesis and replication, mitotic checkpoint control, DNA damage repair and self-renewal, development, and differentiation $^{28,29}$. And E2F1 is the first discovered and most widely studied gene in the E2Fs family. Existing studies have shown that E2F1 is dysregulated in various types of 
human cancers including glioma. Zhi et al. described that ECT2 stabilizes and upregulates the expression of E2F1 through interfering with the deubiquitinating ability of PSMD14 in glioma cells ${ }^{30}$. Moreover, E2F1 has been identified as the target molecule of miRNAs in the regulation of development or cisplatin resistance of glioma cells $^{31,32}$. In ceRNAs, one of the most popular molecular regulatory networks, E2F1 is also considered to be involved as a downstream target gene ${ }^{33,34}$. Given E2F1 as oncogene in glioma and our microarray analysis, we deduced that E2F1 may mediate the effect of CDCA8 in glioma. The interaction between CDCA8 and E2F1 verified by co-IP assay showed that CDCA8 may regulate the progression of glioma through interacting and regulating E2F1. As the potential downstream of CDCA8, knockdown of E2F1 could alleviate or reverse the glioma growth induced by CDCA8 overexpression. Thus, CDCA8/E2F1 axis is responsible for glioma cell growth and migration in vitro or in vivo.

In conclusion, despite the limited numbers of clinical specimens and relatively preliminary mechanism research, we defined CDCA8 as a novel tumor promotor in the development of glioma probably through regulating E2F1. CDCA8 is upregulated in glioma and could be considered as a promising target for cancer therapy based on gene silencing. our study reveals a new underlying mechanism of CDCA8 in glioma. We showed that CDCA8 effectively inhibited cell cycle arrest and cell apoptosis in glioma. Synergized with E2F1, CDCA8 promoted glioma cell proliferation and migration in vitro and vivo. Targeting CDCA8 or E2F1 exerted its antitumor effect in malignant glioma. And clinical data showed that CDCA8 may act as a biomarker for the progression and prognosis of glioma. This study provides a novel mechanistic basis and strategy for clinical application of CDCA8/E2F1 in glioma in the future.

\section{Funding}

This work was supported by National Natural Science Foundation of China (No. 81902554 to X.X. Wang), Postdoctoral Science Foundation of China (No. 2018M641847 to X.X. Wang), Postdoctoral Science Foundation of Heilongjiang Province (No. LBH-Z19190 to X.X. Wang), and Young Medical Talent Foundation of the Frist Affiliated Hospital of Harbin Medical University (HYD2020YQ0018).

\section{Author details}

'Department of Neurosurgery, The First Affiliated Hospital of Harbin Medical University, No. 23 Youzheng Street, Nangang District, 150001 Harbin, Heilongjiang Province, People's Republic of China. ${ }^{2}$ Institute of Brain Science, Harbin Medical University, No. 23 Youzheng Street, Nangang District, 150001 Harbin, Heilongjiang Province, People's Republic of China. ${ }^{3}$ Department of Neurosurgery, TongJi Hospital of TongJi Medical College, Huazhong University of Science and Technology, No.1095 Jie Fang Avenue, Hankou, Wuhan 430030, People's Republic of China. ${ }^{4}$ Department of Neurosurgery, The Sixth Affiliated Hospital of Sun Yat-sen University, No. 26 Erheng Road, Yuan Village, Tianhe District, Guangzhou, People's Republic of China
}

\section{Author contributions}

Y.B. Zhen, H.Q. Zhan, and X.X. Wang conceived, designed, coordinated, and directed this study. X.X. Wang, H.P. Wang, J.J. Xu, and X. Hou performed all molecular and animal experiments. H.P. Wang supervised and performed the statistical analysis in this study. X.X. Wang and H.Q. Zhan wrote this manuscript. Y.B. Zhen and X.X. Wang assessed and modified the manuscript. All authors reviewed and approved the final manuscript.

\section{Conflict of interest}

The authors declare that they have no conflict of interest.

\section{Ethical approval}

All animal experiments conformed to the European Parliament Directive (2010/63/EU) and were approved by the Institutional Animal Care and Use Committee at Harbin Medical University (No. HMUIRB-2008-06) and the Institute of Laboratory Animal Science of China (A5655-01).

\section{Publisher's note}

Springer Nature remains neutral with regard to jurisdictional claims in published maps and institutional affiliations.

Supplementary information The online version contains supplementary material available at https://doi.org/10.1038/s41419-021-03405-4.

Received: 1 August 2020 Revised: 22 December 2020 Accepted: 28 December 2020

Published online: 01 February 2021

\section{References}

1. Ostrom, Q., Gittleman, H., Stetson, L., Virk, S. \& Barnholtz-Sloan, J. Epidemiology of intracranial gliomas. Prog. Neurol. Surg. 30, 1-11 (2018).

2. Siegel, R. L., Miller, K. D. \& Jemal, A. Cancer statistics, 2020. CA Cancer J. Clin. 70, 7-30 (2020).

3. Bush, N. A. O., Chang, S. M. \& Berger, M. S. Current and future strategies for treatment of glioma. Neurosurg. Rev. 40, 1-14 (2017).

4. Rasmussen, B. K. et al. Epidemiology of glioma: clinical characteristics, symptoms, and predictors of glioma patients grade I-IV in the Danish NeuroOncology Registry. J. Neuro-Oncol. 135, 571-579 (2017).

5. Nabors, L. et al. NCCN guidelines insights: central nervous system cancers, version 1.2017. J. Natl Compr. Cancer Netw. 15, 1331-1345 (2017).

6. Masui, K, Kato, Y., Sawada, T., Mischel, P. S. \& Shibata, N. Molecular and genetic determinants of glioma cell invasion. Int. J. Mol. Sci. 18, 2609 (2017).

7. Manini, I. et al. Role of microenvironment in glioma invasion: what we learned from in vitro models. Int. J. Mol. Sci. 19, 147 (2018).

8. Alfonso, J. C. L. et al. The biology and mathematical modelling of glioma invasion: a review. J. R. Soc. Interface 14, 20170490 (2017).

9. van der Horst, A. \& Lens, S. M. A. Cell division: control of the chromosomal passenger complex in time and space. Chromosoma 123, 25-42 (2014).

10. Carmena, M., Wheelock, M., Funabiki, H. \& Earnshaw, W. C. The chromosomal passenger complex (CPC): from easy rider to the godfather of mitosis. Nat. Rev. Mol. Cell Bio. 13, 789-803 (2012).

11. Zhang, C. et al. CDCA8 regulates meiotic spindle assembly and chromosome segregation during human oocyte meiosis. Gene 741, 144495 (2020).

12. Hindriksen, S., Meppelink, A. \& Lens, S. M. A. Functionality of the chromosomal passenger complex in cancer. Biochem. Soc. T 43, 23-32 (2015).

13. Li, F., Aljahdali, I. \& Ling, X. Cancer therapeutics using survivin BIRC5 as a target: what can we do after over two decades of study? J. Exp. Clin. Canc. Res. 38, 368 (2019).

14. Martínez-García, D., Manero-Rupérez, N., Quesada, R., Korrodi-Gregório, L. \& Soto-Cerrato, V. Therapeutic strategies involving survivin inhibition in cancer. Med. Res. Rev. 39, 887-909 (2019).

15. Portella, G., Passaro, C. \& Chieffi, P. Aurora B: a new prognostic marker and therapeutic target in cancer. Curr. Med. Chem. 18, 482-496 (2011).

16. Zhang, Q. et al. Borealin is differentially expressed in ES cells and is essential for the early development of embryonic cells. Mol. Biol. Rep. 36, 603-609 (2009).

17. $\mathrm{Gu}, \mathrm{Y}$. et al. Identification of prognostic genes in kidney renal clear cell carcinoma by RNA-seq data analysis. Mol. Med. Rep. 15, 1661-1667 (2017). 
18. Gusyatiner, O. \& Hegi, M. E. Glioma epigenetics: from subclassification to novel treatment options. Semin. Cancer Biol. 51, 50-58 (2018).

19. Tan, A. C. et al. Management of glioblastoma: state of the art and future directions. CA Cancer J. Clin. 70, 299-312 (2020).

20. van der Waal, M. S., Hengeveld, R. C. C., van der Horst, A. \& Lens, S. M. A. Cell division control by the Chromosomal Passenger Complex. Exp. Cell Res. $\mathbf{3 1 8}$ 1407-1420 (2012)

21. Dai, $C$. et al. Transcriptional activation of human CDCA8 gene regulated by transcription factor NF-Y in embryonic stem cells and cancer cells. J. Biol. Chem. 290, 22423-22434 (2015).

22. Hayama, S. et al. Phosphorylation and activation of cell division cycle associated 8 by aurora kinase B plays a significant role in human lung carcinogenesis. Cancer Res. 67, 4113 (2007)

23. $\mathrm{Ci}, \mathrm{C}$. et al. Overexpression of CDCA8 promotes the malignant progression of cutaneous melanoma and leads to poor prognosis. Int. J. Mol. Med. 43 404-412 (2019).

24. $\mathrm{Bu}$, Y., Shi, L., Yu, D., Liang, Z. \& Li, W. CDCA8 is a key mediator of estrogenstimulated cell proliferation in breast cancer cells. Gene 703, 1-6 (2019).

25. Yu, D., Shi, L., Bu, Y. \& Li, W. Cell division cycle associated 8 is a key regulator of tamoxifen resistance in breast cancer. J. Breast Cancer 22, 237-247 (2019).
26. Wei, W. et al. Identification of key genes involved in the metastasis of clear cell renal cell carcinoma. Oncol. Lett. 17, 4321-4328 (2019).

27. Gao, X. et al. Knockdown of CDCA8 inhibits the proliferation and enhances the apoptosis of bladder cancer cells. PeerJ 8, e9078-e9078 (2020).

28. Johnson, D. \& Schneider-Broussard, R. Role of E2F in cell cycle control and cancer. Front. Biosci. 3, d447-d448 (1998).

29. Kent, L. N. \& Leone, G. The broken cycle: E2F dysfunction in cancer. Nat. Rev. Cancer 19, 326-338 (2019).

30. Zhi, T. et al. ECT2/PSMD14/PTTG1 axis promotes the proliferation of glioma through stabilizing E2F1. Neuro-Oncol. 21, 462-473 (2018).

31. Ying, H. \& Chi, C. Glioma cell proliferation is inhibited by miR-342-3p, miR-377/ E2F1 signaling pathway. Neoplasma 2019, 524-531 (2019).

32. Li, F., Xing, C., Wu, L. \& Xue, F. MiR-205 enhances cisplatin sensitivity of glioma cells by targeting E2F1. Eur. Rev. Med. Pharm. 22, 299-306 (2018).

33. Xia, L., Nie, D., Wang, G., Sun, C. \& Chen, G. FER1L4/miR-372/E2F1 works as a ceRNA system to regulate the proliferation and cell cycle of glioma cells. J. Cell. Mol. Med. 23, 3224-3233 (2019).

34. Li, X., Zhang, H. \& Wu, X. Long noncoding RNA DLX6-AS1 accelerates the glioma carcinogenesis by competing endogenous sponging miR-197-5p to relieve E2F1. Gene 686, 1-7 (2019). 